\title{
Study on the Improved Hub Quality Judgment Algorithm Based on Harmonic Analysis Method
}

\author{
Guang Yang, Tao Feng \& Yongzuo Qin \\ School of Electronic Information Engineering, Changchun University of Science \& Technology \\ Changchun 130022, China \\ Tel: 86-431-8558-2648Ｅ-mail: ygyangguang@126.com
}

Received: May 18, 2011

Accepted: July 23, 2011

doi:10.5539/mas.v5n5p264

\begin{abstract}
The principle of the harmonic analysis method and the its application in the hub runout measurement are introduced in detail in this article, and through the practical measurement data, the geometric meaning of judge the hub quality by the harmonic analysis method is proposed, and the disadvantages of the 8-point coordinate method are analyzed, and the computation method of the Fourier factor is improved, and the reliability to judge the hub quality by the harmonic analysis method is enhanced.
\end{abstract}

Keywords: Runout, Harmonic analysis, 8-point coordinate method

\section{Introduction}

As the key component concerning the safety and comfortableness of vehicle running in high speed, the hub requires higher and higher quality, and the detection is more and more difficult. If the production and manufacturing of hub are not qualified, the tires are easy to be clocked or punctured, which is very dangerous for the motor vehicles running in high speed. Therefore, the hub production enterprises urgently need the meter which can strictly and high-effectively detect the quality of vehicle hub, and need a kind of effective analysis method to judge the hub quality, which should analyze the harmonic data of hub runout. The intelligent hub runout measurement system studied in this article adopts the real-time data acquisition and processing technology, the measuring head movement and measurement rotation of axes closed -loop control technology, and the hub automatic installment dismantlement technology, to realize the automatic detection of hub and the automatic analysis real time, and guarantee the detection precision.

\section{The principle of the hub quality measurement by the harmonic analysis method and the improvement of the Fourier factor algorithm}

The harmonic analysis method is to expand the experiment data recoded automatically as the Fourier series when studying some periodic phenomena, and solve the amplitudes and phases of various harmonics, and change irregular curves into a series of frequency, i.e. the superposition of the sine curves with integer multiple relationships, and these sine curves or sine waves with integer multiple relationship are harmonic waves.

According to the Fourier transform theory, a periodic function can be decomposed as a series of sine wave signal, and a series of sine wave can be superposed as the non-sine wave signals in one period. Because the bub measurement data is the non-sine periodic signal taking the cycle as the unit, and the data acquired when the hub rotates one cycle are the signals in one period. Therefore, it is feasible to analyze the harmonic wave of hub runout from the data acquired when the hub rotates one cycle.

Suppose $f(x)$ is a non-sine periodic function, and its period is T, and its frequency and angular frequency respectively are $f$ and $\omega$. Because the non-sine periodic function in the practice would usually satisfy the Dirichlet condition, it can be unfolded as the Fourier series, i.e.

$$
\begin{aligned}
& y=f(x)=A_{0}+A_{1} \sin \left(x+\varphi_{1}\right)+A_{2} \sin \left(2 x+\varphi_{2}\right)+\mathrm{A}_{3} \sin \left(3 x+\varphi_{3}\right)+\ldots+\mathrm{A}_{\mathrm{n}} \sin \left(\mathrm{n} x+\varphi_{\mathrm{n}}\right)+\ldots \\
& \left.=A_{0}+\sum_{n=1}^{\infty} \sin \left(n x+\varphi_{n}\right) \text { iwhere } \mathrm{n}=1,2,3 \ldots\right)
\end{aligned}
$$

, where, $A_{0}$ is the DC component or the constant component, and others are some sine quantities with different amplitudes, different initial phase angles, and their frequencies have the relationship of integer multiples. The 
item of $A_{1} \sin \left(x+\varphi_{1}\right)$ is the first harmonic or the fundamental harmonic, $A_{1}$ and $\varphi_{1}$ respectively are its amplitude and its initial phase angle, the angular frequency of $A_{2} \sin \left(2 x+\varphi_{2}\right)$ is two times of the angular frequency of the fundamental harmonic $\omega, A_{2} \sin \left(2 x+\varphi_{2}\right)$ is the secondary harmonic, $A_{1}$ and $\varphi_{1}$ respectively are its amplitude and its initial phase angle, and other items respectively are called as the third harmonic, the fourth harmonic, and so on. The fundamental harmonic, the third harmonic, the fifth harmonic... are called by a joint name, the odd harmonic, and the secondary harmonic, the fourth harmonic... are called by a joint name, the even harmonic, and except for the constant component and the fundamental harmonic, other items are called by the higher harmonic. The formula (1) can be transformed as the formula (2).

$$
\begin{aligned}
y=f(x) & =a_{0}+\sum_{n=1}^{\infty} A_{n} \sin \left(n x+\varphi_{n}\right) \\
=a_{0}+\sum_{n=1}^{\infty} a_{n} \cos n x+\sum_{n=1}^{\infty} b_{n} \sin n x & \\
a_{n} & =A_{n} \sin \varphi_{n} \\
b_{n} & =A_{n} \cos \varphi_{n} \\
\text {, where, } \quad & \sqrt{a_{n}^{2}+b_{n}^{2}} \\
A_{n} & \operatorname{arctg}\left(a_{n} / b_{n}\right)
\end{aligned}
$$

The formula (2) is an infinite trigonometric series, and $a_{0}, a_{1}, a_{2} \ldots a_{n}, b_{1}, b_{2} \ldots b_{n}$ is called by Fourier factors, and to solve the amplitudes $A_{n}$ and the phases of various harmonic components, the Fourier factors must be solved first.

However, in most cases, the periodic function is unknown, and only the values of a few points in the function $y=f(x)$ are known. Because $f(x)$ is an infinite series, it only needs to solve finite items according to the demand. If the $n$ 'th harmonic needs to be known, only the $2 n+1$ items need to be taken from the formula. For the computation, the amount of the known points are the integer multiples of 4 , i.e. one period $2 \pi$ is equally divided into $8,12,20,24,36$, and 48 parts, i.e. $8,12,20,24,36$, and 48 pair of data should be computed, which are respectively called by 8-point coordinates method, 12-point coordinates method, 20-point coordinates method, 24-point coordinates method, 36-point coordinates method, and 48-point coordinates method. These methods can respectively solve 1 4'th harmonic, 1 6'th harmonic, 1 10'th harmonic, 1 12'th harmonic, $1 \sim 18^{\prime}$ th harmonic and 1 24'th harmonic.

In the measurement of hub runout, the first harmonic and the second harmonic of hub runout are emphasized. Therefore, the data of the first harmonic and the second harmonic of hub runout need to be computed.

One period $2 \pi$ is equally divided into 8 parts, i.e. taking 8 groups of measurement data in equal interval, which is called by the 8-point coordinate method (seen in Table 1).

By respectively substituting each pair of measurement value $(t, y)$ into the formula (2), $a_{0}, a_{1}, a_{2}, a_{3}, a_{4}, b_{1}, b_{2}, b_{3}, b_{4}$ can be solved.

Supposed that the hub rotates one cycle, the data point quantity acquired by the sensor is 360 , and in virtue of the 8-point coordinate method, only 8 points in Table 1 in the harmonic operation participate in the computation of Fourier factor, and other data are invalid. It is obvious that the Fourier factor computed by the 8-point coordinate method abandoned most data, which will increase the system error undoubtedly. Therefore, the discrete Fourier factor computation method will make all data acquired by the sensor to participate in the operation (seen in Formula 3).

$$
\begin{aligned}
& a_{n}=\frac{1}{\pi} \sum_{i=1}^{N} x_{i} \cos \left(2 \pi \times n \times \frac{i}{N}\right) \\
& b_{n}=\frac{1}{\pi} \sum_{i=1}^{N} x_{i} \sin \left(2 \pi \times n \times \frac{i}{N}\right)
\end{aligned}
$$


, where, $x_{i}$ denotes the $i$ ' th sampling data acquired by the sensor and $N$ is the sampling point quantity of the sensor when the hub rotates one cycle.

After working out the Fourier factor according to the formula (4), the harmonic parameters such as $A_{1}, \varphi_{1}, A_{2}$ and $\varphi_{2}$ can be worked out according to the formula (3), and the first harmonic curve and the second harmonic curve can be figured out according to the formula (5).

$y_{1}=A_{1} \sin \left(2 \pi \omega_{1}+\varphi_{1}\right)$

$y_{2}=A_{2} \sin \left(2 \pi \times 2 \omega_{1}+\varphi_{2}\right)$

, where, $\omega_{1}=\frac{n}{N}$, and $N$ is the sampling point quantity of the sensor when the hub rotates one cycle, and $n$ changes from 1 to $N$.

The harmonic runout of hub is defined by the difference of the positive peak and the negative peak of the harmonic, and from the formula (5), the runouts of the first harmonic and the second harmonic jump $p_{1}$ and jump $p_{2}$ respectively are

jump $=2 \times\left|A_{1}\right|$

jump $p_{2}=2 \times\left|A_{2}\right|$

\section{Geometric meaning of the hub quality judgment by the harmonic analysis data}

After acquiring the measurement data from the measurement sensor, the Fourier factor can be worked out according to the formula (4), and the harmonic form can be figured out according to the formula (5), and the runout of the harmonic can be worked out to judge the hub quality. Figure 1 is the runout data and the harmonic form of the 167-050 hub, where, $A$ is the wave form of the first harmonic, B is the high spot of the first harmonic, $\mathrm{C}$ is the wave form of the original data, $\mathrm{D}$ is the wave form of the second harmonic, and $\mathrm{E}$ is the low spot of the first harmonic.

According to the hub quality technical standards, the judgment thresholds of the first harmonic and the second harmonic respectively are $\eta_{1}$ and $\eta_{2}$, and when the hub runout of the first harmonic jump $p_{1}$ exceeds $\eta_{1}$ and the hub runout of the second harmonic jump $p_{2}$ is less than $\eta_{2}$, i.e.

$$
\left\{\begin{array}{l}
\text { jump } p_{1}>\eta_{1} \\
\text { jump } p_{2}<\eta 2
\end{array}\right.
$$

, the hub is off the center, and as seen in Figure 2 (a), the dotted line is the qualified hub, $\mathrm{O}$ is the center of a circle of the qualified hub, and the full line is the detected hub, and $\mathrm{O}_{1}$ is the center of a circle of the detected hub.

When the hub runout of the first harmonic jump $p_{1}$ is less than $\eta_{1}$ and the hub runout of the second harmonic jump $p_{2}$ exceeds $\eta_{2}$, i.e.

$\left\{\begin{array}{l}\text { jump } p_{1}<\eta_{1} \\ \text { jump } p_{2}>\eta 2\end{array}\right.$

, the hub presents the ellipse shape, and as seen in Figure 2 (b), the dotted line is the qualified hub, $\mathrm{O}$ is the center of a circle of the qualified hub, and the full line is the detected hub, $F_{1}$ and $F_{2}$ are two focuses of the detected ellipse hub.

In the practice, whether the runout data of the first harmonic or the runout data of the second harmonic could not satisfy the technical standard, the hub is judged as unqualified.

\section{Conclusions}

By improved Fourier factor computation method, the study in this article strengthens the reliability of detecting the hub quality by the harmonic method, enhances the detection precision, and provides reliable data for the judgment of hub quality. Through observing the harmonic analysis figure, testing personnel can adjust the 
manufacturing precision of the lathe according to the harmonic runout, and at the same time, the quality control department also can definitely judge whether the hub runout exceeds the standard.

\section{References}

Yu, Xiaohui. (2008). Design of the Measurement and Control System of the Vehicle Hub Runout Measuring Machine Based on DSP. Changchun: Master's Thesis of Changchun University of Science \& Technology. April of 2008 .

Zhou, Fuchen et al. (2000). Technical Manual of Mechanical Manufacturing Measurement \& Detection. Beijing: China Machine Press.

Table 1. 8-point coordinates method

\begin{tabular}{|c|c|c|c|c|c|c|c|c|}
\hline$x$ & $0^{\circ}$ & $45^{\circ}$ & $90^{\circ}$ & $135^{\circ}$ & $180^{\circ}$ & $225^{\circ}$ & $270^{\circ}$ & $315^{\circ}$ \\
\hline$y$ & $y_{0}$ & $y_{1}$ & $y_{2}$ & $y_{3}$ & $y_{4}$ & $y_{5}$ & $y_{6}$ & $y_{7}$ \\
\hline
\end{tabular}

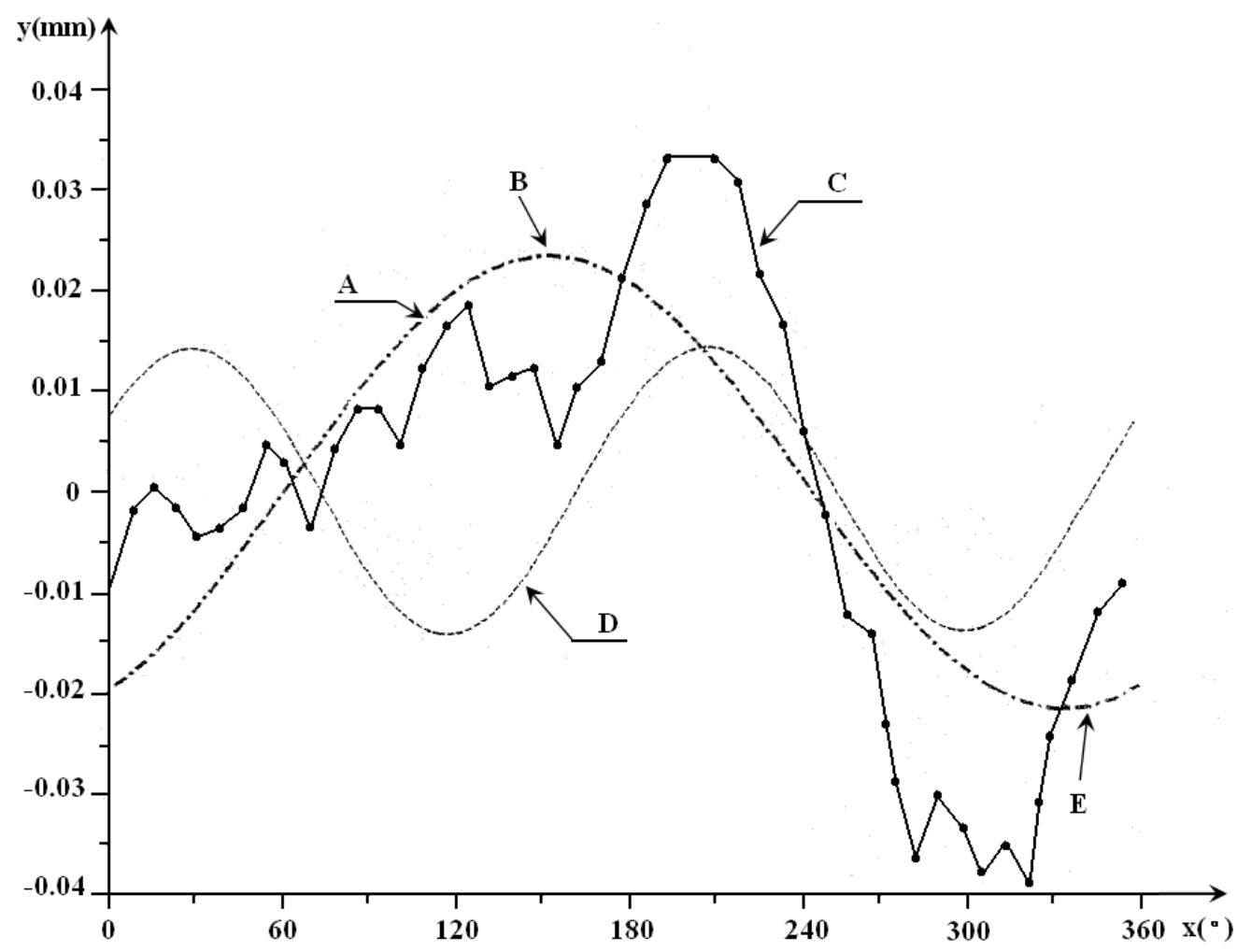

Figure 1. Original Data and Harmonic Data Wave Form 


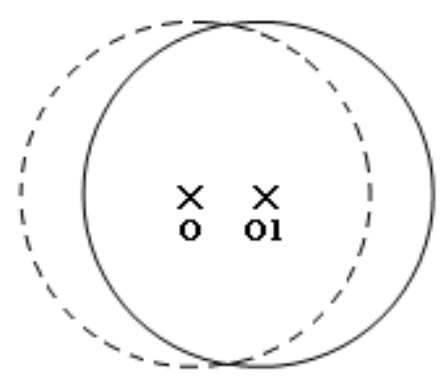

(a) first harmonic

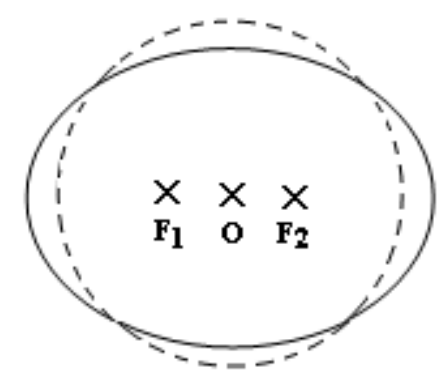

(b) secondary harmonic

Figure 2. Geometric Meaning of the Hub Harmonic Wave Runou 